\title{
PRODUÇÃO DE PECTINASES EM CULTIVO SUBMERSO DE Aspergillus oryzae SEM LIMITAÇÃO DE OXIGÊNIO
}

\author{
L. MENEGHEL, C. ROSSI, G. P. REIS, C. REGINATTO, \\ E. MALVESSI, M. M. SILVEIRA \\ Universidade de Caxias do Sul, Instituto de Biotecnologia \\ E-mail para contato: $\underline{\text { lmscatharina@ucs.br }}$
}

\begin{abstract}
RESUMO - O suprimento de oxigênio em cultivos de microrganismos aeróbios é fundamental para garantir adequados crescimento e produção de metabólitos, afetando diretamente o desempenho do processo. Na produção de pectinases fúngicas, a alta viscosidade do meio, conferida pela pectina, e as intensas modificações reológicas, provocadas pelo crescimento celular, prejudicam a mistura e a transferência de oxigênio. Neste trabalho, a pectina (indutor enzimático) foi adicionada ao cultivo após a fase de intenso crescimento celular, permitindo o estudo da produção de pectinases por Aspergillus oryzae em meio líquido sem limitação de oxigênio. Verificou-se que, apesar de o crescimento celular ter sido semelhante ao do ensaio controle, a não limitação de oxigênio permitiu a obtenção de atividade enzimática $(120 \mathrm{U} / \mathrm{mL})$, praticamente quatro vezes maior que a do controle $(43 \mathrm{U} / \mathrm{mL})$. Os resultados indicam que a condução do processo sem limitação de oxigênio resulta em ganho substancial na produção de pectinases.
\end{abstract}

\section{INTRODUÇÃO}

Os microrganismos aeróbios e os anaeróbios facultativos, quando utilizados em processos biotecnológicos, necessitam de uma disponibilidade ilimitada de oxigênio dissolvido no meio para garantir o adequado crescimento. $\mathrm{O}$ consumo de oxigênio dissolvido também influencia a manutenção celular e a produção de metabólitos. Quando em escassez, afeta diretamente o desempenho do processo (Garcia-Ochoa \& Gomez, 2009).

O suprimento de oxigênio em cultivos submersos é promovido pela operação de dissolução de oxigênio contido na fase gasosa (normalmente ar) para a fase líquida, para que esteja disponível para o consumo microbiano. O fato de o oxigênio ser pouco solúvel em água torna a extensão dos processos aeróbios e a obtenção de produtos dependentes da capacidade de se transferir oxigênio para a fase líquida (Schmidell et al., 2001).

As pectinases, enzimas utilizadas em diferentes setores comerciais, em especial na indústria de alimentos (Reid e Ricard, 2000; Sandri et al., 2013) podem ser produzidas por muitas espécies de fungos filamentosos, microrganismos particularmente exigentes quanto à transferência de oxigênio uma vez que podem apresentar formação de pellets ou filamentos livres. (Kashyap et al., 2001). 
Em processos com microrganismos aeróbios, é esperado, em princípio, que um suprimento não limitado de oxigênio - ou seja, a manutenção da concentração de oxigênio dissolvido sempre acima de seu valor crítico - seja suficiente para garantir a eficiência do processo. Entretanto, Zetelaki-Horvath \& Vas (1981) relatam que, apesar de o crescimento de $A$. niger ter sido favorecido a uma velocidade de transferência de oxigênio (OTR) de $100 \mathrm{mmol} / \mathrm{L} / \mathrm{h}$, as atividades de pectina liase e endo-polimetilgalacturonase foram aumentadas com OTR de $60 \mathrm{mmol} / \mathrm{L} / \mathrm{h}$, enquanto que para endopoligalacturonase, a OTR de $49 \mathrm{mmol} / \mathrm{L} / \mathrm{h}$ foi a melhor condição. Também, Lai et al. (2005) e Li et al. (2008) verificaram que a limitação temporária de oxigênio durante o cultivo tem efeito positivo sobre a obtenção de metabólitos de fungos do gênero Aspergillus.

Este trabalho teve por objetivo estudar a produção pectinases por $A$. oryzae em meio líquido sem limitação de oxigênio em comparação com uma condição controle em que houve limitação no fornecimento de $\mathrm{O}_{2}$ ao cultivo.

\section{MATERIAIS E MÉTODOS}

O microrganismo utilizado foi A. oryzae IPT-301, cedido pelo Instituto de Pesquisas Tecnológicas de São Paulo. O meio de cultivo empregado foi o descrito por Meneghel (2013), composto de extrato de farelo de trigo, glicose, pectina, extrato de levedura e sais. Os cultivos foram conduzidos em biorreator de bancada Biostat B (B. Braun Biotech), com 3,5L de meio, frequência de agitadores e fluxo de ar iniciais de $300 \mathrm{rpm}$ e $0,57 \mathrm{vm}$, a $28^{\circ} \mathrm{C}$, por $160 \mathrm{~h}$. O biorreator foi programado de forma a manter-se a concentração de oxigênio dissolvido em $30 \%$ da saturação, pela variação automática da frequência dos agitadores e/ou fluxo de ar. No ensaio controle, a frequência de agitadores e o fluxo de ar foram mantidos constantes em $480 \mathrm{rpm}$ e $0,42 \mathrm{vvm}$. O indutor enzimático - pectina cítrica - foi adicionado ao meio somente após $24 \mathrm{~h}$ de cultivo e o $\mathrm{pH}$ foi inicialmente corrigido em 4,0 e mantido neste valor até $24 \mathrm{~h}$, em seguida reduzido para 2,7 pela adição de $\mathrm{HCl}$ e controlado até o final do cultivo.

O crescimento celular foi avaliado por gravimetria e os açúcares redutores totais (ART), pelo método descrito por Bitmann (1974), que prevê a hidrólise ácida, adaptado para amostras isentas de sólidos suspensos. O método de medição de ART não é influenciado pela pectina.

A atividade de pectinases totais foi avaliada pelo método descrito por Malvessi (2000). Este método é baseado na medida da redução da viscosidade de uma solução padrão de pectina a $0,63 \%(\mathrm{~m} / \mathrm{v})$ submetida à ação de enzimas pectinolíticas que rompem as ligações glicosídicas internas da cadeia de ácido poligalacturônico.

A velocidade específica de crescimento celular $\left(\mu_{\mathrm{X}}\right)$ foi determinada a partir das concentrações celulares medidas nas amostras, durante a fase exponencial do cultivo.

$\mathrm{O}$ fator de produção específica $\left(\mathrm{Y}_{\mathrm{P} / \mathrm{X}}\right)$ foi calculado a partir dos máximos valores de atividade enzimática $\left(\mathrm{P}_{\max }\right)$ e concentração de biomassa $\left(\mathrm{X}_{\max }\right)$ obtidos no cultivo.

Os fatores de conversão de substrato em produto $\left(\mathrm{Y}_{\mathrm{P} / \mathrm{S}}\right)$ e em células $\left(\mathrm{Y}_{\mathrm{X} / \mathrm{S}}\right)$ foram calculados a partir dos máximos valores de atividade enzimática $\left(\mathrm{P}_{\max }\right)$ e concentração de 
biomassa $\left(\mathrm{X}_{\max }\right)$ obtidos no cultivo, e da concentração de substrato consumido até o tempo em que ocorreram os picos.

\section{RESULTADOS E DISCUSSÃO}

Neste estudo, procurou-se proporcionar ao microrganismo a possibilidade de realizar a respiração na máxima velocidade específica em cada momento do processo. Neste sentido, a concentração de oxigênio dissolvido foi mantida acima da concentração crítica, para que o oxigênio não se tornasse limitante. A concentração crítica de oxigênio em meio aquosos para A. oryzae é de cerca de 0,02mmol/L (Bailey \& Ollis, 1986; Schmidell et al., 2001). Considerando que a concentração de oxigênio dissolvido em água, na altitude local, é de $0,213 \mathrm{mmol} / \mathrm{L}$, e que a presença dos nutrientes do meio reduz este valor, a concentração crítica de oxigênio para $A$. oryzae, nas condições de estudo é de cerca de $10 \%$ da saturação. Assim, optou-se por manter-se a concentração de oxigênio em $30 \%$ da saturação, admitindo-se que, neste valor, tinha-se a garantia de ilimitada disponibilidade de $\mathrm{O}_{2}$ para o cultivo.

Os resultados gerais do Ensaio O30 (oxigênio dissolvido mantido automaticamente em torno de 30\% da saturação), assim como do Ensaio Controle (com limitação de oxigênio dissolvido), são resumidos na Tabela 1. Na Figura 1, são ilustrados os perfis cinéticos observados em $\mathrm{O} 30$.

Tabela 1 - Resultados gerais de cultivo de Aspergillus oryzae em biorreator de bancada sem limitação de oxigênio (O30) em comparação com o controle.

\begin{tabular}{|c|c|c|}
\hline & $\mathbf{O 3 0}$ & Controle \\
\hline$X_{\max }(g / L)$ & 6,8 & 6,3 \\
\hline $\mathbf{t}_{\mathbf{X}, \max }(\mathrm{h})$ & 36 & 36 \\
\hline$\mu_{X, \max }\left(h^{-1}\right)$ & 0,30 & 0,18 \\
\hline$t_{\mu X, \max }(h)$ & 17,5 & 19 \\
\hline$S_{\text {cons }}(g / L)$ & 11,2 & 10,8 \\
\hline$P_{\max }(U / m L)$ & 120 & 43 \\
\hline $\mathbf{t}_{\mathrm{P}, \max }(\mathbf{h})$ & 120 & 140 \\
\hline $\mathbf{Y}_{\mathbf{P} / \mathbf{X}}(\mathrm{U} / \mathbf{m g})$ & 17,6 & 6,82 \\
\hline$Y_{X / S}(g / g)$ & 0,62 & 0,58 \\
\hline$Y_{P / S}(U / m g)$ & 10,7 & 3,98 \\
\hline$p_{V}(\mathrm{U} / \mathrm{mL} / \mathbf{h})$ & 1,0 & 0,31 \\
\hline $\mathrm{pH}_{\min }$ & 2,7 & 2,7 \\
\hline $\mathrm{pH}_{\text {final }}$ & 2,7 & 2,7 \\
\hline
\end{tabular}

$\mathrm{X}_{\max }$ - máxima concentração de biomassa; $\mathrm{t}_{\mathrm{X}, \max }-$ tempo em que ocorreu $\mathrm{X}_{\max } ; \mu_{\mathrm{X}, \max }$ - máxima velocidade específica de crescimento celular; $\mathrm{t}_{\mu \mathrm{X}, \max }-$ tempo em que ocorreu $\mu_{\mathrm{X}, \max } ; \mathrm{S}_{\text {cons }}$ - consumo total de ART ; $\mathrm{P}_{\max }-$ máxima atividade de pectinases; $t_{\mathrm{P}, \max }$ - tempo em que ocorreu $\mathrm{P}_{\max } ; \mathrm{Y}_{\mathrm{P} / \mathrm{X}}$ - fator de produção específica; $\mathrm{Y}_{\mathrm{X} / \mathrm{S}}$ - fator de conversão de substrato em células; $\mathrm{Y}_{\mathrm{P} / \mathrm{S}}$ - fator de conversão de substrato em pectinases; $\mathrm{p}_{\mathrm{V}}$ - produtividade volumétrica; $\mathrm{pH}_{\text {min }}-$ mínimo valor de $\mathrm{pH}$ do cultivo; $\mathrm{pH}_{\text {final }}$ - valor final de $\mathrm{pH}$ do cultivo.

As concentrações de biomassa alcançadas nos Ensaios O30 e controle foram similares, podendo ter sido favorecidas pela condição de $\mathrm{pH}$. No entanto, em O30, a velocidade 
específica de crescimento celular foi superior. Aparentemente, a não limitação de oxigênio dissolvido para o fungo, acelerou o crescimento celular.

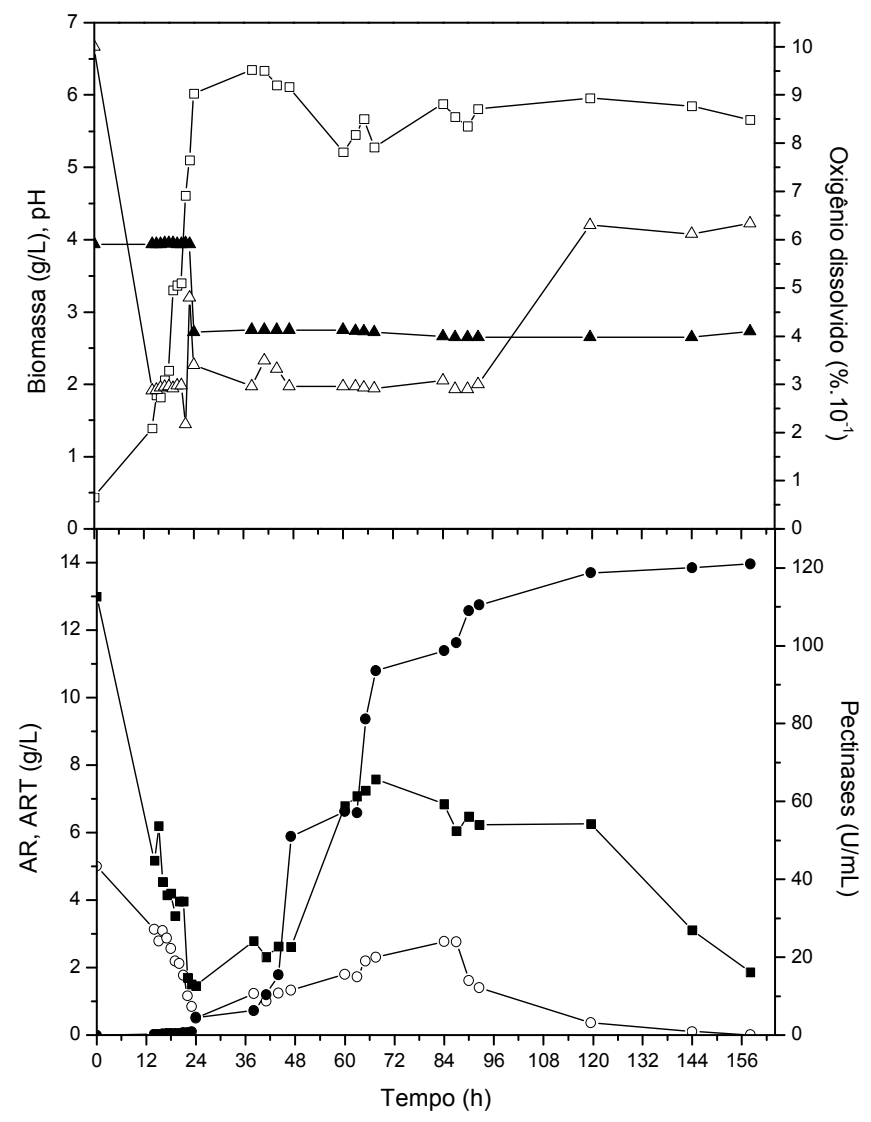

Figura 1 - Variação das concentrações de biomassa, açúcares redutores, açúcares redutores totais e oxigênio dissolvido, da atividade de pectinase e do $\mathrm{pH}$ em cultivo de Aspergillus oryzae, em biorreator de bancada, com concentração de oxigênio mantido em mínimo de $30 \%$

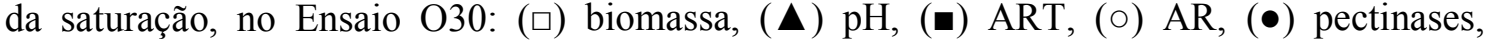
$(\Delta)$ oxigênio dissolvido.

As condições de consumo de substrato deste ensaio podem estar relacionadas aos valores de $\mathrm{pH}$ proporcionados pela estratégia adotada para o controle deste parâmetro. Após a redução forçada para o valor de 2,7 , em $24 \mathrm{~h}$, o consumo de substrato foi mais lento. Além disso, observou-se um sensível aumento de AR e ART em torno de $36 \mathrm{~h}$ de processo (Figura 1), possivelmente como resultado da presença de substâncias redutoras liberadas pela hidrólise do indutor, adicionado ao cultivo em $24 \mathrm{~h}$

A atividade enzimática no Ensaio $\mathrm{O} 30$ começou a ser verificada a partir de $36 \mathrm{~h}$. No entanto, a disponibilidade de substrato (pectina) teria possibilitado a liberação de alta quantidade de enzimas $(120 \mathrm{U} / \mathrm{mL})$ em relação ao controle $(43 \mathrm{U} / \mathrm{mL})$, atingindo pico de atividade em $120 \mathrm{~h}, 20 \mathrm{~h}$ antes que o ensaio controle. 
Em O30, o máximo teor de oxigênio dissolvido ao fim do cultivo foi de $65 \%$ da saturação. Este dado, aliado à manutenção da concentração de biomassa até o final do processo, indica que, nesta condição, foi possível manter a viabilidade celular por mais tempo. No caso, a maior concentração de células viáveis em O30 teria proporcionado os altos valores de atividade enzimática obtidos neste ensaio.

Ressalte-se que, o fator de produção específica obtido em O30 foi 2,6 vezes superior que o controle, indicando que maior número de unidades de pectinases resultaram de cada unidade de massa de biomassa formada. Considerando que, conforme observado na Figura 1, os perfis cinéticos de crescimento celular e formação de enzimas não são associados, altos valores de $\mathrm{Y}_{\mathrm{P} / \mathrm{X}}$ indicam a possibilidade de obtenção de expressiva atividade enzimática mesmo quando a biomassa resultante do processo for reduzida.

Além do fator de produção específica, a produtividade volumétrica alcançada em O30 foi também superior, em cerca de três vezes (Tabela 1), indicando aumento no rendimento global do processo em relação ao controle.

Em O30, foi possível obter-se atividade enzimática máxima de $120 \mathrm{U} / \mathrm{mL}$, praticamente três vezes maior que a do controle $(43 \mathrm{U} / \mathrm{mL})$. Ao contrário do que foi observado por ZetelákiHorváth \& Vas (1981), Lai et al. (2005), Li et al. (2008) e Bettin (2010), a não limitação de oxigênio dissolvido favoreceu o crescimento celular e, também, a produção de pectinases.

\section{CONCLUSÕES}

Os resultados confirmam que o suprimento de oxigênio afeta decisivamente a capacidade de produção de pectinases por $A$. oryzae e indicam que a condução do processo sem limitação de oxigênio resulta em ganho substancial na produção de pectinases.

\section{AGRADECIMENTOS}

Ao CNPq, à CAPES, à FAPERGS e à UCS pelo apoio na realização deste trabalho.

\section{REFEREÊNCIAS BIBLIOGRÁFICAS}

BAILEY, J.E.; OLLIS, D.F. Biochem. Eng. Fund. Singapore: McGraw-Hill, Inc. pp. 984, 1986.

BETTIN, F. Cinética de crescimento e produção e lacases do fungo Pleurotus sajor-caju PS2001 (Fr.) Singer em processo submerso em biorreator com agitação mecânica. Tese de doutorado. Instituto de Biotecnologia, Universidade de Caxias do Sul, 2010.

BITTMAN, R. Analysis of reducing sugars in breakfast cereal and other foods. J. Chem. Education. v. 51, p. 49, 1974.

GARCIA-OCHOA, F.; GOMEZ, E. Bioreactor scale-up and oxygen transfer rate in microbial processes: an overview. Biotechnol. Adv. v. 27, p. 153-176, 2009. 
KASHYAP, D.R.; VOHRA, P.K.; CHOPRA, S.; TEWARI, R. Applications of pectinases in the commercial sector: a review. Bioresour. Technol. v. 77, p. 215-227, 2001.

MALVESSI, E. Estudo de produção de poligalacturonases por Aspergillus oryzae em processo submerso. Dissertação de mestrado. Instituto de Biotecnologia, Universidade de Caxias do Sul, 2000.

MENEGHEL, L. Avaliação da produção de pectinases por Aspergillus oryzae IPT-301 em processo submerso. Dissertação de mestrado. Instituto de Biotecnologia, Universidade de Caxias do Sul, 2013.

REID, I.; RICARD, M. Pectinase in paper making, solving retention problems in mechanical pulps bleached with hydrogen peroxide. Enzyme Microb. Technol. v. 12, p. 115-123, 2000.

SANDRI, I.G.; LORENZONI, C.M.T.; FONTANA, R.C.; SILVEIRA, M.M. Use of pectinases produced by a new strain of Aspergillus niger for the enzymatic treatment of apple and blueberry juice. LWT - Food Sci. Technol. v. 51, p. 469-475, 2013.

SCHMIDELL, W.; LIMA, U.A.; AQUARONE, E.; BORZANI, W. Biotecnologia industrial. Vol. 2 - Engenharia Bioquímica. São Paulo: Edgard Blucher Ltda. pp. 541, 2001. 